\title{
Contraindications for Tattooing
}

\author{
Nicolas Kluger \\ University of Helsinki and Helsinki University Central Hospital, Dermatology, Helsinki, Finland
}

\begin{abstract}
Tattooing is getting increasingly popular among the young. However, not everyone is suited to getting tattooed. Indeed, it is not rare for patients with a chronic skin disease or another systemic condition to be eager to get a tattoo. They perceive tattooing as a harmless, risk-free procedure. Therefore, some patients may not seek medical advice before the procedure. Some also fear a judgmental approach by their physician, who may try to discourage them. Lastly, the tattooist does not have either the training or the education to properly advise a customer about his/her condition. Therefore, it is important that any physician be able to provide adequate counselling regarding the possibility of getting tattooed and under which conditions. Even though an exhaustive list is impossible to address, the main issues include chronic skin disorders, pigmented lesions of the skin, (congenital) heart disease, immunosuppressive diseases and treatments, blood clotting disorders, and pregnancy/breastfeeding.

(C) 2015 S. Karger AG, Basel
\end{abstract}

The contraindications for tattooing are by far one of the most important questions regarding customers' safety. This question is also paradoxically the most underrepresented in terms of medical publications. In our experience, too many physicians tend to quickly judge and contraindicate tattooing for patients, without any real data to support such a position. From our point of view, there is no strict contraindication to getting a tattoo done; however, there is definitely a certain number of situations in which individuals who want to get a tattoo should first seek medical advice to determine whether or not they can get one and, if so, under which conditions they can get it. In our daily experience, we are often contacted by professional tattooists or patients, usually with common skin conditions such as psoriasis, atopic dermatitis, or eczema, to find out whether the patients' skin is suited to being tattooed. However, we have also had to answer questions about more uncommon situations and patients with rarer 
conditions, such as cirrhosis, renal graft, angiomas, the Sutton phenomenon, von Willebrand disease, or even pseudoxanthoma elasticum or xeroderma pigmentosum!

Therefore, it is impossible to be fully exhaustive regarding all skin and systemic conditions to consider when deciding whether or not someone can get a tattoo. This chapter intends to summarize the most frequent situations and questions that a physician may have to deal with. From our perspective, every physician should know about these situations and be able to deliver correct and honest information to a patient who wants to get a tattoo.

\section{Skin Diseases}

\section{Chronic Skin Disorders}

The main risk for patients with chronic skin disorders when getting tattooed, which is often a matter of concern for them, is the development of the very same lesion at the site of the tattoo, e.g. the Köbner phenomenon [1]. Advising our patients with chronic skin disorders who want to get a tattoo is challenging. With the multitude of possible skin disorders, no one can predict whether a specific condition may lead to Köbnerization. The risk of Köbnerization for one disease varies from one patient to another. Meanwhile, some patients use tattooing as a way to camouflage skin lesions (scars, extended vitiligo, etc.) and/or to regain control of their body and cope with their (disfiguring) disease (fig. 1,2). The potential beneficial effects of tattooing should not be neglected. Therefore, advice about disease should be given on a case-by-case basis, especially if the 'well-being' benefits are underlined.

According to the Boyd-Nelder classification, only three diseases do Köbnerize (psoriasis, lichen planus, and vitiligo) [2, 3] (fig. 3, 4). However, by experience, the Köbner phenomenon may complicate any dermatological disease, which may be localized to a site of previous trau-

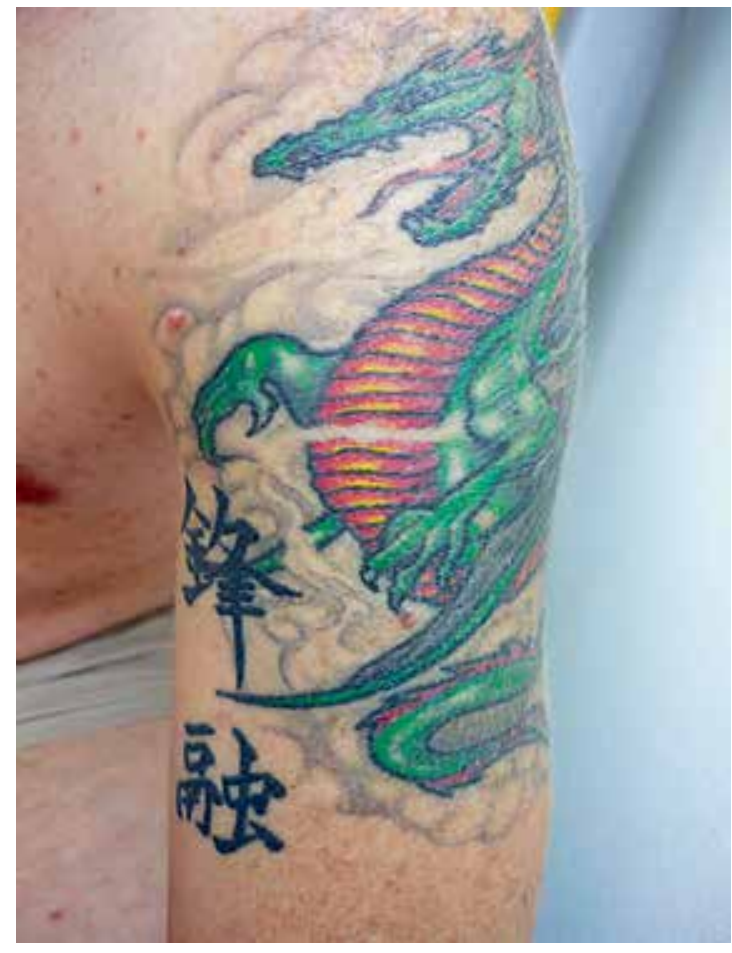

Fig. 1. Tattoo on a young patient with neurofibromatosis type 1 .

ma. It is therefore not surprising that a wide number of dermatologic conditions have been described as arising on tattoos, even though such cases are usually anecdotal (table 1) [1].

It is not uncommon for us to be contacted by customers, patients, or tattooists about a condition and questioned about the 'risk' of developing a reaction to a tattoo. Overall, from our point of view, chronic skin disorders per se are not strict contraindications for tattooing. However, honest information should be given to the customer about his/her risk so that he/she can decide freely to get a tattoo or not. First, the risk of the Köbner phenomenon is rather unpredictable in terms of to whom it will occur and at which point. The Köbner phenomenon may occur between days and years afterward. Moreover, during the whole life of the bearer, there is a risk that someday, during a flare of the skin disease, a rash may occur on the 
Fig. 2. Tattoo on the wrist to hide self-inflicted scarifications.

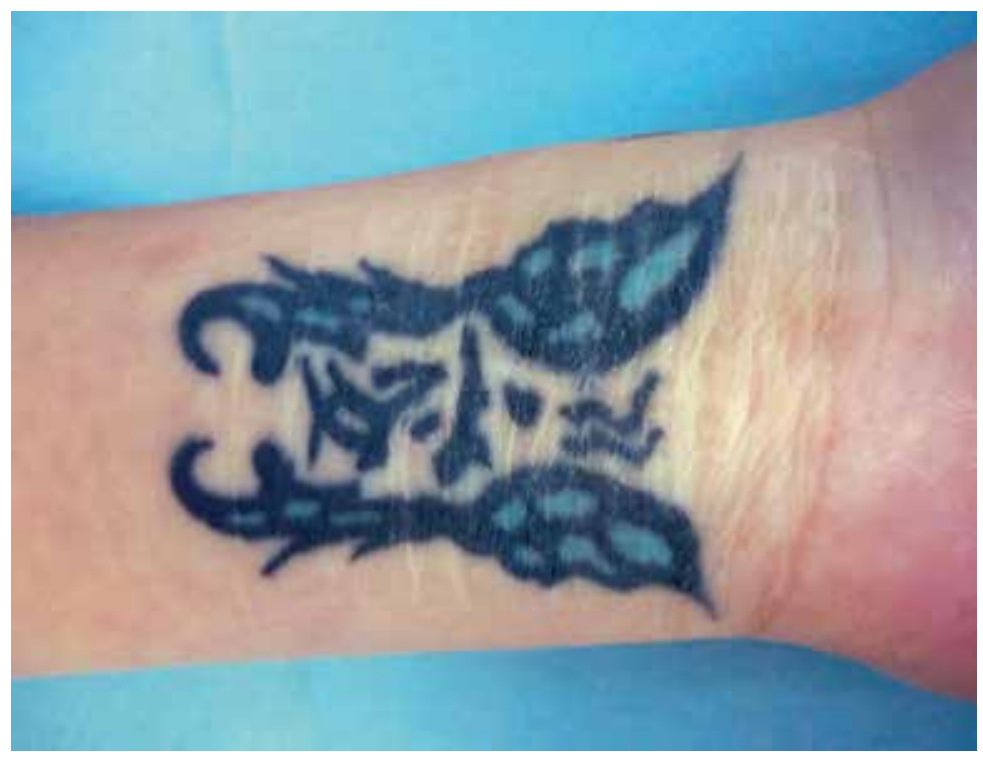

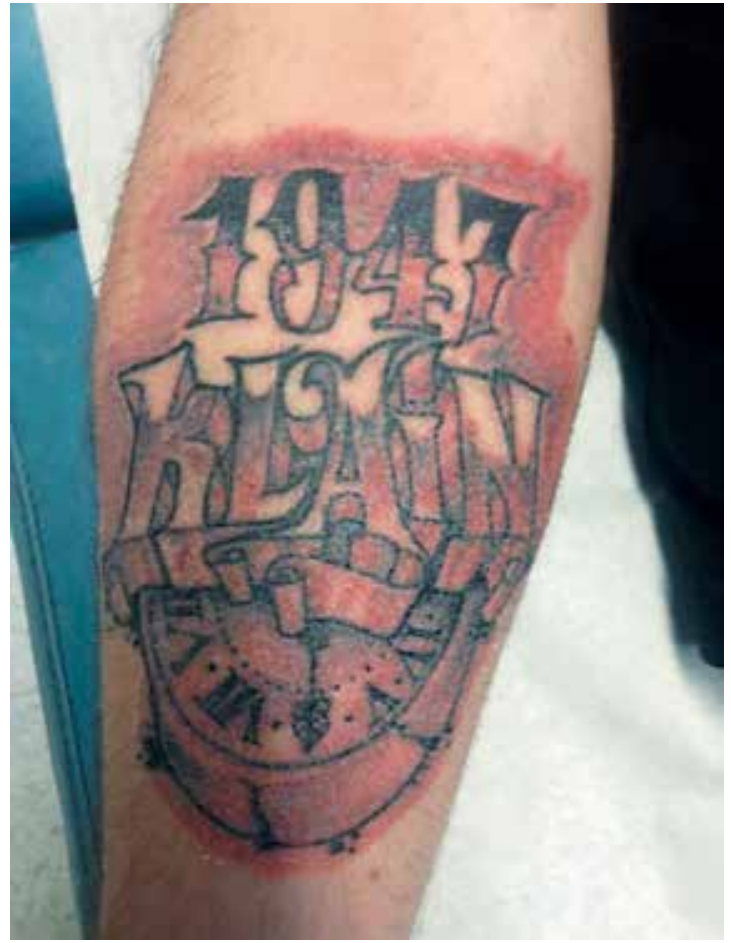

Fig. 3. Psoriasis patch on a recent tattoo in a patient with psoriasis guttata (courtesy of Dr. Levy-Rameau, Montpellier, France). tattoo. The Köbner phenomenon is rarely 'complete', involving the whole tattoo; it may occur only on a part of the tattoo. Patients with a past history of the Köbner phenomenon on trauma sites, scars, or tattoos are more at risk. For instance, it is known that the Köbner phenomenon is an 'all-or-none' phenomenon in psoriatic patients (patients have it or do not). The risk of Köbnerization is higher if the disease is currently active. Therefore, tattooing should be delayed until the skin is clear of any (or any new) lesions and is quiescent. Based on the previous reasons, we simply discourage the so-called 'tattoo test spot on a hidden skin area' of a patient to test whether or not a patient is prone to Köbnerization. There is absolute uncertainty about the criteria for positivity or negativity (the tattoo ink quantity used in the test, the tattoo color, the delay of positivity, etc.). Not only would negative results be falsely reassuring but there is also a risk of sensitizing the customer to a tattoo ingredient. Even though tattoos have been used to cover mucosal vitiligo, tattooing on the edge of a vitiligo patch may trigger extension of the vitiligo. Moreover, the final visible result of a colored tattoo is not the same on 
Fig. 4. Vitiligo patch restricted to a part of a tattoo (courtesy of Dr. Giacchero, Nice, France).

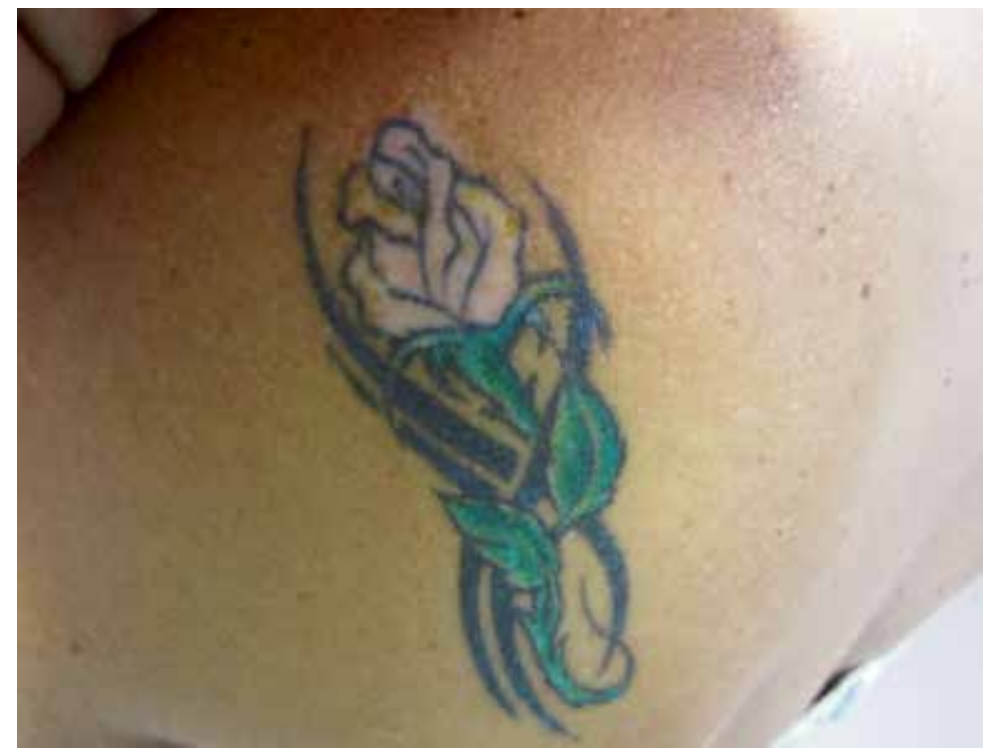

Table 1. Nonexhaustive list of skin disorders that have been reported on tattoos [1]

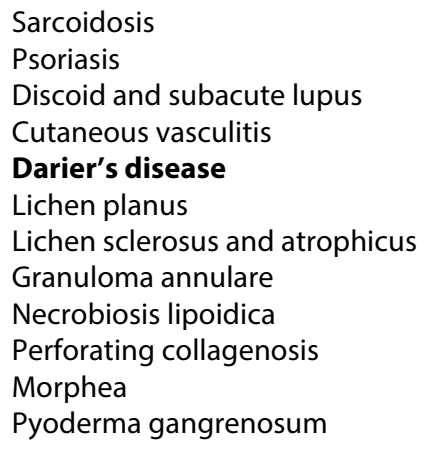

vitiliginous skin as on normal skin due to the lack of melanin. The physician should take into account that the treatment may include immunosuppressive therapy, such as for instance tumor necrosis factor (TNF)-alpha blockers for psoriasis, which leads to other potential issues (cf. below). In the case of skin lesions occurring on a tattoo, the treatments are exactly the same as if the disorder occurred on plain skin.
However, not every skin condition requires caution. For instance, whereas keloids are a strict contraindication for body piercings, they are not a contraindication for professional tattooing. Indeed, keloids on tattoos have been reported in specific situations: (i) tattoo burns after improper use of a laser for hair removal (on a tattooed area) [4] and (ii) ethnic tattoo scarifications in Africa. Similarly, one may think intuitively that tattooing a patient with epidermolysis bullosa may lead to blisters. This is not the case in our experience, with a few patients having undergone tattooing with no blisters. Cutaneous sarcoidosis can manifest with sarcoid papules and nodules on a tattoo [5]. However, rather than a complication, the tattoo acts as an indicator of the disease rather than a trigger. Patients with a past history of sarcoidosis are not contraindicated for tattooing, taking into account possible systemic immunosuppressive treatments (cf. below). Systematic screening for sarcoidosis before tattooing in customers with a family history of sarcoidosis is overexaggerated and has no relevance. 


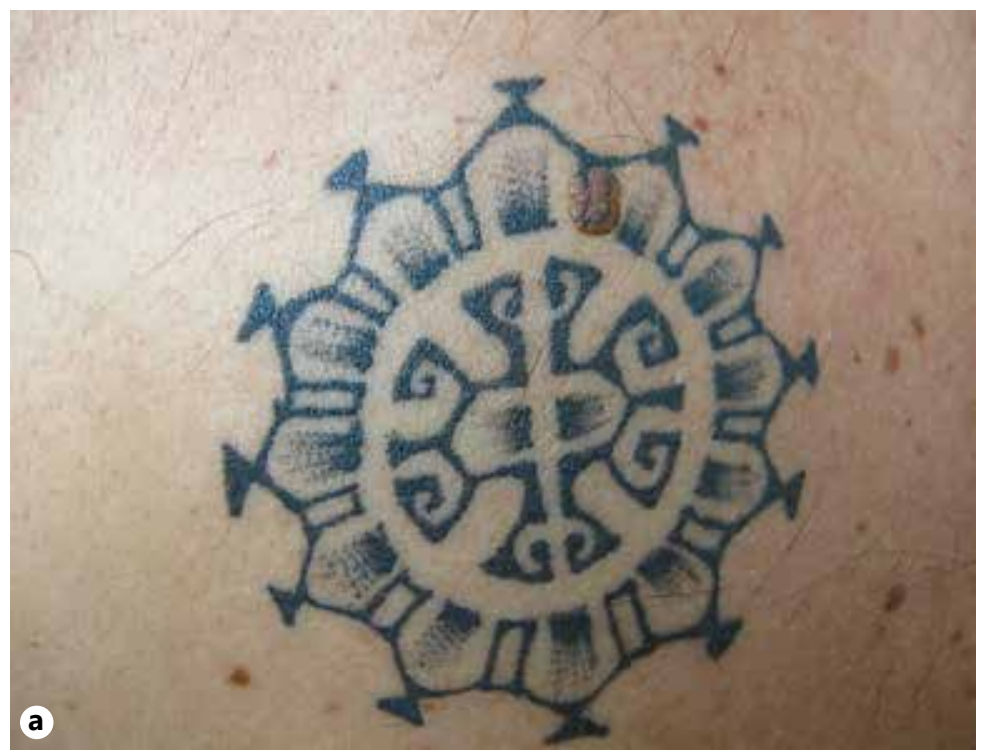

Fig. 5. Tattoo on the back with drawings performed on a naevus (a) and its aspects on dermatoscopy (b) (courtesy of Dr. Comte, Paris, France).

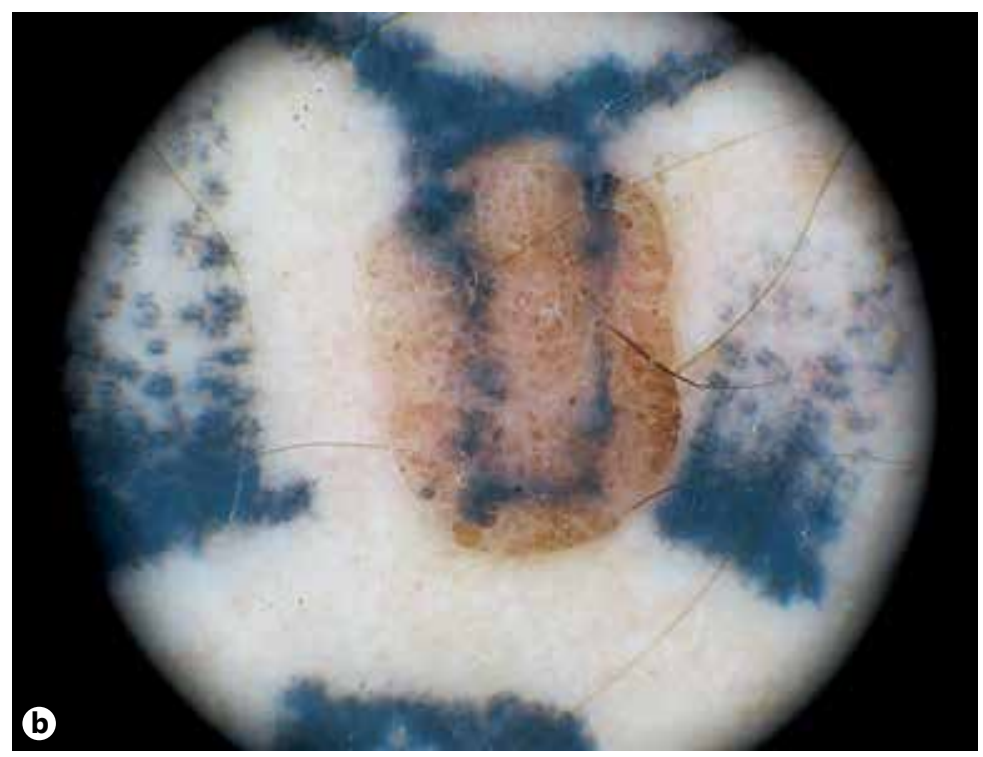

Naevus and Other Pigmented Lesions

Professional tattooists are generally aware that they should not tattoo on any pigmented lesion. However, experience shows that this is not always the case, even nowadays (fig. 5).

After tattooing, a traumatized tattooed naevus may be altered and display clinical changes and atypical histological features that can raise con- cern about malignancy, such as pagetoid spread, cytologic atypia, and dermal mitosis [6]. Changes may even be delayed for years afterward due to a possible autograft phenomenon [7]. Even though a whether a single or persistent traumatic event can be a causative factor for melanoma remains debated, a clinical change in a naevus on a tattoo should prompt its immediate and complete 


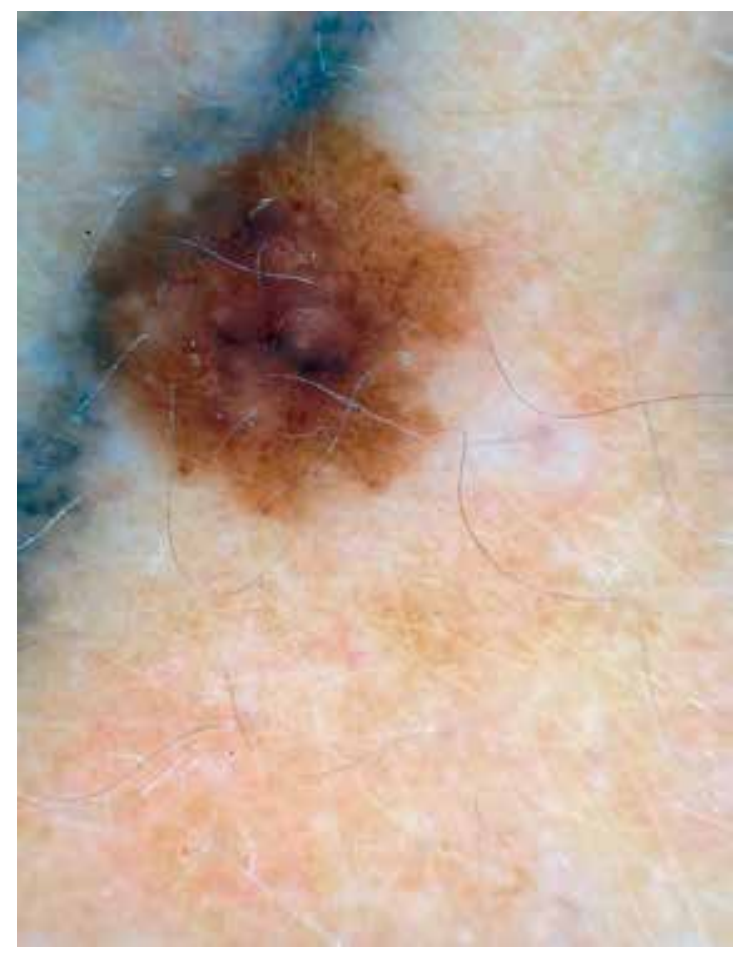

Fig. 6. Dermoscopic aspect of a naevus on a tattoo. Notice the excess pigmentation on the upper part due to the dermal pigments.

excision, along with a microscopic analysis [6]. The correct assessment of the patterns of a pigmented lesion by dermatoscopy is hindered by the presence of exogenous colored particles [810] (fig. 6).

As a general rule, no naevus or any pigmented lesion should be tattooed (fig. 7). A sufficient amount of space should be left if a naevus finds itself in the way of the tattoo. There is no rule for how much space should be given, but a minimum of $0.5-1 \mathrm{~cm}$ (no less) is necessary, as a nevus can still extend with time. The tattooist should avoid tattooing 'just on the edge' (fig. 8). We recommend that patients with a personal history of melanoma avoid tattoos because of the theoretical risk of a second melanoma arising on a tattoo, whose diagnosis would be delayed [8]. However, a 'small' and 'light' tattoo with bright colors on a

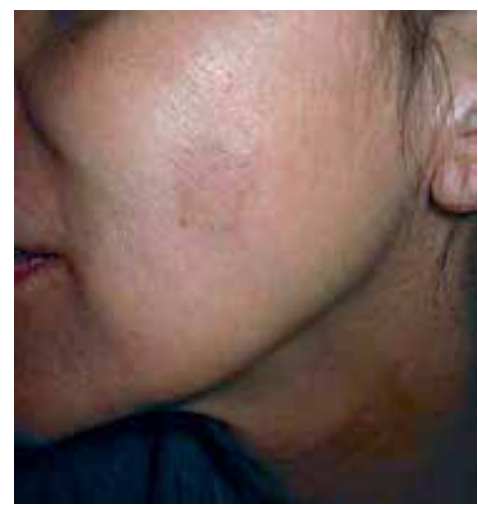

Fig. 7. Facial lentigo that was tattooed to camouflage it (courtesy of Dr. Colonna, Porto-Vecchio, France).

carefully selected area could be allowed. Young patients with a familial history of melanoma, numerous naevi, or atypical mole syndrome who are considering getting a tattoo should have a specialized consultation with a dermatologist, with a full check-up, before getting tattooed. The best location for a tattoo could be discussed, and atypical lesions could be removed before tattooing. A patient should avoid getting a tattoo in an area with lots of naevi. Customers and tattooists must know that covering a naevus with tattoos will, on the one hand, disturb its clinical surveillance and, on the other hand, possibly trigger clinical changes that will necessitate surgical excision and useless alteration of the tattoo. These customers should either avoid being tattooed on a location with a naevus and/or insist on having a tattoo whose drawings will spare the pigmented lesions.

\section{Systemic Diseases}

\section{Heart Conditions}

Infective endocarditis (IE) after tattooing is rather uncommon, especially when compared with piercing [11]. The prevalence of congenital heart disease is estimated to be $1 \%$ overall in the general population. It is likely that a similar 
Fig. 8. Example of a tattoo sparing naevi, with sufficient space, but also artistically incorporating the naevi into the tattoo (courtesy of Dr. Werber, Toulouse, France).

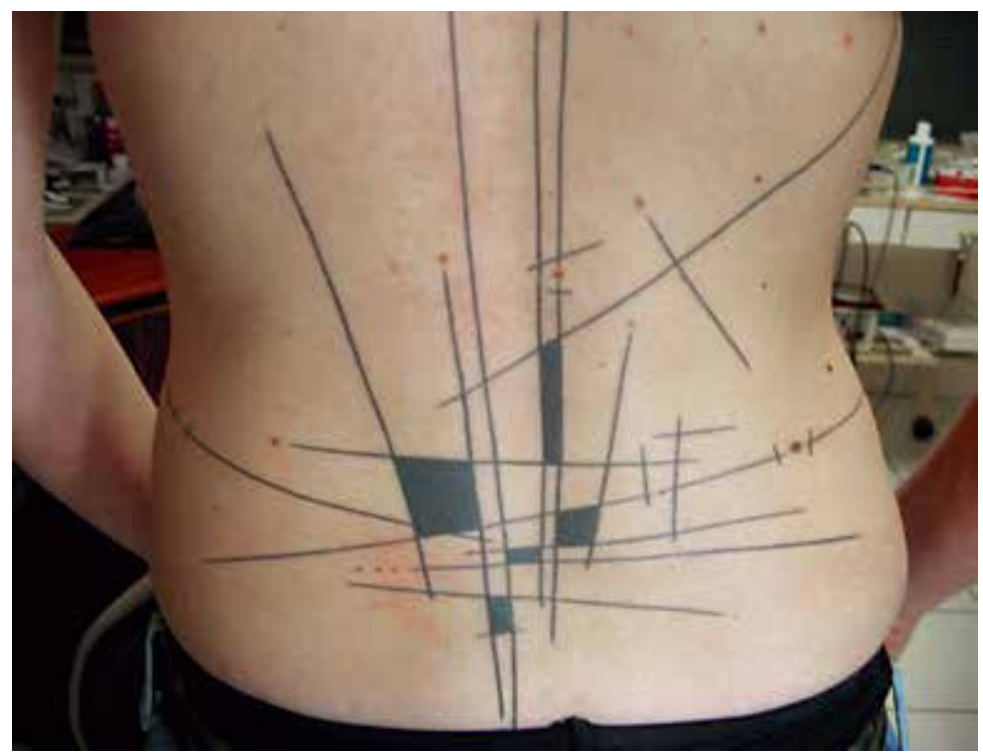

percentage of customers presenting to a body-art professional would have a (diagnosed or undiagnosed) congenital heart disease [12, 13]. In 1999, Cetta et al. reported that a minority of patients with congenital heart disease $(5 \%, 8 / 152)$ had received a tattoo without antibiotic use and had no infectious adverse events [14]. However, a few cases of IE after tattooing have been reported among patients with congenital heart disease during the past few years [15-19]. In three of those cases, the patients did not seek advice before tattooing $[15,17,19]$, while in one, the patient was unaware of his condition [18].

IE is associated with bacteremia, potential dissemination, and multiple septic emboli [18]. In the context of tattooing, IE is associated with Staphylococcus species inoculation $[15,17,19]$, which occurs either during the tattooing procedure because of a lack of hygiene, a mistake during the disinfection procedures, or the use of contaminated material, or possibly during the healing phase because of a lack of hygiene/proper after-care [12]. It is important, though, to rule out any other possible cause of IE before considering the tattoo as the culprit (dental procedure, intra- venous drug intake, etc.). In the absence of another identified cause, a recent tattoo can be considered as the cause [19]. IE leads to a need for intravenous antibiotics and valvular replacement $[17,18]$. To our knowledge, heart transplantation has not been reported thus far after tattoo-related IE.

Patients with congenital heart diseases may not perceive the risk of IE during tattooing [15]. They may be reluctant to share their wish of getting a tattoo with their physician, as the physician may not approve based on experience [14].

As a congenital heart disease may go unnoticed $[12,18]$, any unusual symptom occurring within weeks after tattooing (fever, chills, malaise, night sweats, or dyspnea) should prompt examination for a heart murmur and possible IE. An asymptomatic tattoo, devoid of any local sign of infection, should not rule out the diagnosis of IE $[18,19]$.

We recommend the following to prevent IE after tattooing $[12,13]$ :

(i) Patients with congenital heart diseases should be educated about the risk of IE during tattooing and body piercing procedures. 
(ii) Any (future) customer with a known history of a cardiac murmur, even if only mentioned in childhood or some distant past history, should delay the procedure and seek for medical advice, followed, if necessary, by further specialized explorations.

(iii) Any patient with a known congenital heart disease should warn the general practitioner/cardiologist about the procedure, ask for updated data, and discuss possible prophylactic antibiotics.

(iv) The tattooist should be warned of the heart condition by the customer, and the procedure should be performed with the maximum asepsis (for instance, using fresh, new bottles of ink for tattooing).

The role of antibiotic prophylaxis in such invasive nonmedical procedures remains debated $[13,14]$. Therefore, prophylaxis needs to be discussed on a case-by-case basis with the physician in charge. Lastly, the intake of antithrombotic treatment for a heart condition and the risk of bleeding during tattooing are other issues that should be addressed (cf. below).

\section{Diabetes}

Diabetes is one of the most common diseases worldwide. Type 1 diabetes is the most common type of diabetes in children and adolescents, but type 2 diabetes is increasing among the young [20]. It is therefore not uncommon that a diabetic patient may be interested in getting a tattoo. As a matter of fact, medical alert tattooing, e.g. a medical tattoo identification, is getting popular, at least among some patients with diabetes, in order to alert others about their condition in case of emergency [21]. There are no specific guidelines regarding the tattooing procedure in diabetic customers. According to the stage of evolution of the diabetes, the patients may be exposed to an increased risk of infection and delay of healing. To date, reports of tattoo-related complications in diabetic patients are quite rare. To the best of our knowledge, only a case of erysipelas of the lower limb was reported in a patient with type 2 diabetes [22]. It is not clear to what extent diabetes was directly involved in this complication.

Naturally, out of caution, we suggest that any diabetic patient should warn his/her physician about his/her wish for a tattoo. The tattooist should also be aware of such a condition. Diabetes should be controlled by the time of the tattooing procedure, as assessed by hemoglobin Alc, and the skin area where the tattoo will be applied should be devoid of any skin lesion. Some authors suggest avoiding tattooing the feet or the lower limbs because of the risk of diabetes-induced neurovascular disease [23]. We think that this contraindication seems rather excessive and should be discussed on a case-by-case basis, according to the presence or absence of micro- or macroangiopathy. Moreover, as the tattoo session may last several hours, the customer should take measures to prevent possible hypoglycemia.

Lastly, granuloma annulare and necrobiosis $l i$ poidica are rare dermatologic conditions that have been sometimes associated with diabetes. As a matter of fact, several cases of granuloma annulare and necrobiosis lipoidica on tattoos have been reported in nondiabetic patients [24-26]. Diabetes could be an additional risk factor for such local complications on tattoos.

\section{Inherited and Acquired Blood Disorders}

Transient bleeding during tattooing inevitably occurs as the needles puncture the papillary dermis. During the procedure, the tattooist wipes out the suffusion of blood, and the bleeding usually stops rapidly. As the tattoo is wrapped with a plastic film for a few hours up to overnight, it is not unusual to find secretions and drops of bloods when removing the film. A discrete purpura may sometimes be seen under the tattooed area as red cells deposit in the dermis. Extensive ecchymosis under the tattoo is rare but possible and can raise concern for the customer if he/she has not been warned [27]. The evolution is spontaneously favorable, with resorption of the ecchymosis, and 
the skin color will follow the local bilirubin production. No cataclysmic or life-threatening bleeding or compressive hematoma has been reported after 'professional' tattooing to date.

However, young patients with inherited blood disorders (IBDs, such as hemophilia or von Willebrand disease) and also older patients with acquired blood disorders (such as thrombopenia) or receiving an anticoagulation or an antithrombotic treatment (heparin, anti-vitamin $\mathrm{K}$, or simply aspirin) may want to get a tattoo. As for congenital heart diseases, these patients do not always correctly perceive the potential risk of bleeding. They may neglect, either unconsciously or on purpose, to mention their condition to the tattooist. Moreover, the tattooist may not ask about or consider such a risk. Khair et al. recently showed that having an IBD did not prevent half of the respondents in their study from getting tattooed or pierced [28]. It is clear that there are some patients with an IBD or an acquired blood disorder who undergo tattooing. We have the experience of a young patient with von Willebrand disease who underwent two tattoos, with no complication.

On the Internet, it is widely known and accepted that tattoos are contraindicated for patients with blood disorders. Thus, some patients are being refused tattoos 'systematically' because of an IBD. From our point of view, such a strict position should be moderated, and systematically refusing tattooing to such patients remains debatable. The patients may feel 'different' because they (think that they) cannot get tattooed. We know by experience that, at least for some individuals, tattooing has a positive effect in terms of ego bolstering and is part of a coping strategy as a way to regain control of their own body, as for diabetes, as stressed above [21].

Nowadays, the following is therefore important: (i) health care providers (nurses and physicians) should understand the medical issues associated with tattooing, have a nonjudgmental approach, and be able to correctly inform patients about the risks; (ii) patients should discuss their wish to get tattooed with their physician; (iii) tattoo artists should systematically delay the procedure and refer such patients to their physicians, and (iv) these patients must be considered for prophylactic treatment before such a procedure [29].

For patients presenting with antithrombotic coagulation, the reason why they are receiving such treatment, with a mechanical valve and cardiopathies among the indications, should be investigated, and then, the risk of endocarditis should be discussed. Temporary withdrawal of the treatment for the tattoo session should be discussed with the physician in charge [29].

\section{Immunosuppressive Conditions and Therapies}

Nowadays, the risk of infections after tattooing has greatly improved, thanks to the education of tattooists and their conformity to standard procedures of hygiene and asepsis. Infections mainly occur due to a lack of such procedures, especially in cases of amateur, home-made tattoos or if performed by unlicensed tattooist [30]. Infection may also occur in cases of severe immunodeficiency. Several years ago, Tendas et al. reported the case of a young, 24-year-old Italian who developed an ecthyma gangrenosum on a recent tattoo during an acute myeloid leukemia relapse [31]. We did not find any case of severe infection after tattooing in patients under immunosuppressive therapies [32]. We have the personal experience of young patients who were tattooed while under immunosuppressive therapies (oral corticosteroids, methotrexate, TNF-alpha blockers). According to the patients, the anti-TNF-alpha drugs were responsible for increased fatigue, diminished stamina after the session, a delay in tattoo healing, and secondary cutaneous infection after a session due to an after-care error. Moreover, one of our patient developed psoriasis on her tattoo, as a TNF-alpha blocker was given for this indication [32]. 
Patients with human immunodeficiency virus are exposed to the same complications as any other individuals whose immunity is at acceptable levels. A tattoo reaction has been reported during immune restoration in a single case [33]. Moreover, patients with human immunodeficiency virus specifically develop leishmaniasis on tattoos due to the tropism of this parasite for macrophages [34, 35].

When an immunosuppressive treatment is initiated in a young patient, the physician should inquire about the patient's potential wish for a tattoo. Increased awareness should be encouraged among those who already have tattoos, as they may be more likely to get a new one and may not perceive the risk. The level of immunosuppression related to the therapies, the disease itself, and the potential comorbidities (such as diabetes) have to be taken into account. A nonjudgmental approach is mandatory to allow a better understanding of the situation by the patients and better adherence to potential contraindications. The reasons for contraindications should be explained to the patient, stressing that they are temporary and related to the risk of severe infections. A tattoo can always be reconsidered when the treatment is withdrawn or at a maintenance level. In contrast, it remains reasonable to delay/avoid any tattoo when the disease is active and the treatments are currently at high dosages [32]. Systematically advising patients against a tattoo under mild immunosuppressive treatment is not supported by experience or data in the literature [36]. Lastly, patients may develop other complications on tattoos, such as the Köbner phenomenon on a tattooed area, if the immunosuppressive treatment is given again for a skin condition (psoriasis, sarcoidosis, chronic cutaneous lupus, etc.). The role of etanercept in an anecdotal case of a granulomatous tattoo reaction has been speculated [37].

If permission for a tattoo is granted, the patient should choose a tattooist who respects the rules of asepsis and hygiene in his/her parlor. The tattooist should know about the condition of the patient so that he/she can take the maximum precautions to avoid potential inoculation, such as using new tattoo bottles for the patient, performing a shorter session, paying attention to any unusual local symptoms before a new session, and consulting the patient immediately in the case of such symptoms [32].

\section{Pregnancy and Breastfeeding}

To date, no complication regarding the infant has been reported after a mother has undergone tattoo session(s) during pregnancy or breastfeeding [38]. Tattooists, parturient patients, and breastfeeding mothers are unlikely to perform/get tattoos during that specific period. However, some of us have experience with a pregnant woman or a breastfeeding mother insisting on getting tattoos [39]. Moreover, parturient women may undergo sessions at the very beginning of their pregnancy, while still unaware of it. Others may omit mentioning that they are breastfeeding when presenting to the tattooist. Overall, the prevalence of such situations is totally unknown. For the mother, the complications are the same as for any other individuals getting a tattoo. For the fetus, the risks of transmission of infection and toxic chemicals through the placenta and the milk have never been evaluated. Inoculation of microorganisms and local infection after tattooing and inoculation of blood-transmitted diseases remains low in parlors respecting strict hygiene procedures. Potential bacterial dissemination, although always possible, is exceptional. Moreover, maternal bacterial infections are rarely transmitted to their infants through breast milk. Temporary cessation of breastfeeding may be proposed for a limited time. Antibiotics are usually not a contraindication for breastfeeding but have to be chosen carefully during pregnancy. Some of the chemicals found in tattoo inks (heavy metals, amines, etc.) can be transferred through the human placenta [39]. There are few data regarding breast milk, and the potential systemic distribution of tattoo ingredi- 
ents or by-products in the circulation and therefore possibly through the placenta during pregnancy or in the milk is not known. However, the existence of nanoparticles $(<100 \mathrm{~nm})$ in tattoo inks reopens this question. The risks of undergoing tattooing during pregnancy or breastfeeding are unclear and rather theoretical. However, because a tattoo can always be performed afterward, we suggest delaying any tattooing procedure until the end of pregnancy/breastfeeding. Additionally, the risk to fetal development in heavily tattooed mothers is not known either. The risk re- lated to tattooing as a potential environmental occult source of toxic chemicals for the fetus should be thoroughly investigated. Of note, we have the experience of 25 tattooed women in France who are also professional tattooists who had 36 pregnancies within the context of their professional activities. The favorable outcomes of the pregnancies in our series are rather reassuring in terms of the possible diffusion of tattoo ink particles through the placenta (data unpublished, under submission).

\section{References}

1 Kluger N: Cutaneous complications related to permanent decorative tattooing. Expert Rev Clin Immunol 2010;6:363371.

2 Weiss G, Shemer A, Trau H: The Koebner phenomenon: review of the literature. J Eur Acad Dermatol Venereol 2002;16:241-248.

3 Rubin AI, Stiller MJ: A listing of skin conditions exhibiting the koebner and pseudo-koebner phenomena with eliciting stimuli. J Cutan Med Surg 2002;6: 29-34.

4 Kluger N: Sarcoidosis on tattoos: a review of the literature from 1939 to 2011. Sarcoidosis Vasc Diffuse Lung Dis 2013; 30:86-102.

5 Kluger N, Hakimi S, Del Giudice P: Keloid occurring in a tattoo after laser hair removal. Acta Derm Venereol 2009;89: 334-335.

6 Kluger N, Catala D, Thibaut I: Naevus and tattooing: a matter of concern. J Eur Acad Dermatol Venereol 2008;22:767768.

7 Persechino S, Caperchi C, Bartolazzi A: Melanoma mimicry on a tattoo: an autograft hypothesis. J Am Acad Dermatol 2007;57:S122-S123.

8 Kluger N, Thomas L: The dragon with atypical mole syndrome. Arch Dermatol 2008;144:948-949.

\$9 Gall N, Bröcker EB, Becker JC: Particularities in managing melanoma patients with tattoos: case report and review of the literature. J Dtsch Dermatol Ges 2007;5:1120-1121.
10 Pohl L, Kaiser K, Raulin C: Pitfalls and recommendations in cases of laser removal of decorative tattoos with pigmented lesions: case report and review of the literature. JAMA Dermatol 2013; 149:1087-1089.

11 Armstrong ML, DeBoer S, Cetta F: Infective endocarditis after body art: a review of the literature and concerns. J Adolesc Health 2008;43:217-225.

12 Kluger N: Bacterial endocarditis and body art: suggestions for an active prevention. Int J Cardiol 2009;136:112-113.

13 Tse DM, Khan S, Clarke S: Bacterial endocarditis and body art: active prevention or antibiotic prophylaxis. Int J Cardiol 2010;139:297-298.

14 Cetta F, Graham LC, Lichtenberg RC, Warnes CA: Piercing and tattooing in patients with congenital heart disease: patient and physician perspectives. J Adolesc Health 1999;24:160-162.

15 Satchithananda DK, Walsh J, Schofield PM: Bacterial endocarditis following repeated tattooing. Heart 2001;85:1112.

16 Shebani SO, Miles HF, Simmons P, Stickley J, De Giovanni JV: Awareness of the risk of endocarditis associated with tattooing and body piercing among patients with congenital heart disease and paediatric cardiologists in the United Kingdom. Arch Dis Child 2007;92:10131014.

17 Tse D, Khan S, Clarke S: Bacterial endocarditis complicating body art. Int J Cardiol 2009; 133:e28-e29.
18 Akkus NI, Mina GS, Fereidoon S, Rajpal S: Tattooing complicated by multivalvular bacterial endocarditis. Herz 2014;39: 349-351.

19 Orton CM, Norrington K, Alam H, Alonso-Gonzalez R, Gatzoulis M: The danger of wearing your heart on your sleeve. Int J Cardiol 2014;175:e6-e7.

20 Canivell S, Gomis R: Diagnosis and classification of autoimmune diabetes mellitus. Autoimmun Rev 2014;13:403-407.

21 Kluger N, Aldasouqi S: The motivations and benefits of medical alert tattoos in patients with diabetes. Endocr Pract 2013;19:373-376.

22 Ahluwalia R, Mills A, Cuthbertson D: An 'Avatar' infection: associated cellulitis in a type 2 diabetes patient following decorative tattooing. Practical Diabetes 2011;28:292.

23 Glassy CM, Glassy MS, Aldasouqi S: Tattooing: medical uses and problems. Cleve Clin J Med 2012;79:761-770.

24 Bethune GC, Miller RA, Murray SJ, Walsh NM: A novel inflammatory reaction in a tattoo: challenge. Am J Dermatopathol 2011;33:740-741, 749.

25 Kluger N, Godenèche J, Vermeulen C: Granuloma annulare within the red dye of a tattoo. J Dermatol 2012;39:191-193.

26 Wood A, Hamilton SA, Wallace WA, Biswas A: Necrobiotic granulomatous tattooreaction: report of an unusual case showing features of both necrobiosis lipoidica and granuloma annulare patterns. Am J Dermatopathol 2014;36: e152-e155. 
27 Kluger N: Acute complications of tattooing presenting in the ED. Am J Emerg Med 2012;30:2055-2063.

28 Khair K, Holland M, Pollard D: The experience of girls and young women with inherited bleeding disorders. Haemophilia 2013;19:e276-e281.

29 Kluger N: Tattooing, piercing and inherited coagulation disorders. Haemophilia 2013;19:e358-e359.

30 Kluger N, Saarinen K: Aspergillus fumigatus infection on a home-made tattoo. Br J Dermatol 2014;170:1373-1375.

-31 Tendas A, Niscola P, Barbati R, Abruzzese E, Cuppelli L, Giovannini M, Scaramucci L, Fratoni S, Ales M, Neri B, Morino L, Dentamaro T, De Fabritiis P: Tattoo related pyoderma/ectyma gangrenous as presenting feature of relapsed acute myeloid leukaemia: an exceptionally rare observation. Injury 2011;42:546-547.
32 Kluger N: Tattooing and piercing: an underestimated issue for immunocompromised patients? Presse Med 2013

33 Silvestre JF, Albares MP, Ramón R, Botella R: Cutaneous intolerance to tattoos in a patient with human immunodeficiency virus: a manifestation of the immune restoration syndrome. Arch Dermatol 2001;137:669-670.

34 Colebunders R, Depraetere K, Verstraeten T, Lambert J, Hauben E, Van Marck E, Maurer T, Bañuls AL, Dujardin JC: Unusual cutaneous lesions in two patients with visceral leishmaniasis and HIV infection. J Am Acad Dermatol 1999;41:847-850.
35 Kluger N: Leishmaniasis in Spanish tattoos. Enferm Infecc Microbiol Clin 2010; 28:667.

36 O'Connor MB, Phelan MJ: Should rheumatology patients on immunosuppressive medications be advised against getting tattoos? Ir Med J 2012;105:124.

37 Bachmeyer C, Blum L, Petitjean B, Kemiche F, Pertuiset E: Granulomatous tattoo reaction in a patient treated with etanercept. J Eur Acad Dermatol Venereol 2007;21:550-552.

38 Kluger N: Body art and pregnancy. Eur J Obstet Gynecol Reprod Biol 2010;153: 3-7.

39 Kluger N: Can a mother get a tattoo during pregnancy or while breastfeeding? Eur J Obstet Gynecol Reprod Biol 2012; 161:234-235.

Nicolas Kluger

Departments of Dermatology, Allergology and Venereology, Institute of Clinical Medicine University of Helsinki, Skin and Allergies Hospital, Helsinki University Central Hospital Meilahdentie 2, PO Box 160

FIN-00029 HUS, Helsinki (Finland)

E-Mail nicolaskluger@yahoo.fr 